\title{
Promoting human embryonic stem cell renewal or differentiation by modulating Wnt signal and culture conditions
}

\author{
Liuhong Cai ${ }^{1,2, *}$, Zhaohui Ye ${ }^{1,3, *}$, Betty Ying Zhou ${ }^{1,5}$, Prashant Mali ${ }^{1,4}$, Canquan Zhou ${ }^{2}$, Linzhao Cheng ${ }^{1,3}$ \\ ${ }^{I}$ The Institute for Cell Engineering, Department of Gynecology \& Obstetrics, the Johns Hopkins University School of Medicine, Bal- \\ timore, MD 21205, USA; ${ }^{2}$ Reproductive Medicine Center, The 1st Affiliated Hospital of Zhongshan University, Guangzhou 510089, \\ China; ${ }^{3}$ Graduate Immunology program, the Johns Hopkins University School of Medicine, Baltimore, MD 21205, USA, ${ }^{4}$ Graduate \\ Program of Biomedical Engineering, the Johns Hopkins University School of Medicine, Baltimore, MD 21205, USA
}

We previously showed that Wnt3a could stimulate human embryonic stem (hES) cell proliferation and affect cell fate determination. In the absence of feeder cell-derived factors, hES cells cultured under a feeder-free condition survived and proliferated poorly. Adding recombinant Wnt3a in the absence of feeder cell derived-factors stimulated hES cell proliferation but also differentiation. In the present study, we further extended our analysis to other Wnt ligands such as Wnt1 and Wnt5a. While Wnt1 displayed a similar effect on hES cells as Wnt3a, Wnt5a had little effect in this system. Wnt3a and Wnt1 enhanced proliferation of undifferentiated hES cells when feeder-derived self-renewal factors and $\mathrm{bFGF}$ are also present. To explore the possibility to promote the proliferation of undifferentiated $\mathrm{hES}$ cells by activating the Wnt signaling, we overexpressed Wnt3a or Wnt1 gene in immortalized human adult fibroblast (HAFi) cells that are superior in supporting long-term growth of undifferentiated hES cells than primary mouse embryonic fibroblasts. HAFi cells with or without a Wnt transgene can be propagated indefinitely. Over-expression of the Wnt3a gene significantly enhanced the ability of HAFi feeder cells to support the undifferentiated growth of 3 different hES cell lines we tested. Co-expression of three commonly-used drug selection genes in Wnt3a-overpressing HAFi cells further enabled us to select rare hES clones after stable transfection or transduction. These immortalized engineered feeder cells (W3R) that co-express growth-promoting genes such as Wnt3a and three drug selection genes should empower us to efficiently make genetic modified hES cell lines for basic and translational research.

Cell Research (2007) 17:62-72. doi:10.1038/sj.cr.7310138; published online 9 January 2007

Keywords: WNT, human embryonic stem cells, stem cell renewal, stem cell differentiation, transgene

\section{Introduction}

Pluripotent hES cell lines offer unprecedented opportunities to investigate human cell biology and to develop novel cell-based therapies $[1,2]$. Initially, primary mouse

\footnotetext{
*These two authors contributed equally to this work.

${ }^{5}$ Current address: Whitehead Institute for Biomedical Research, Cambridge, MA, 02142, USA

Correspondence: Linzhao Cheng

Stem Cell Program, Institute for Cell Engineering, The Johns Hopkins University School of Medicine, Broadway Research Building, Room 747, 733 North Broadway, Baltimore, MD 21205, USA

Tel: 1-410-614-6958; Fax: 1-443-287-5611

E-mail: 1cheng@welch.jhu.edu

Received 26 September 2006; revised 18 December 2006; accepted 22 December 2006; published online 9 January 2007
}

embryonic fibroblasts (pMEFs) were used as feeder cells to establish and propagate hES cell lines. Several groups have subsequently found that selected human cells of fetal or adult origins can substitute pMEFs in supporting the prolonged growth of undifferentiated hES cells [3-7]. The expanded hES cells cultured on these supportive human feeder cells after many passages continue to form compact colonies of cells expressing undifferentiated markers such as alkaline phosphatase (AP). These studies also found, however, that many human cell types were not supportive: AP positive $\left(\mathrm{AP}^{+}\right)$compact colonies of undifferentiated hES cells disappeared after 2 passages. The pace of downregulation in the expression of $\mathrm{AP}$ and other undifferentiated markers in differentiating hES cells is much slower 
than mouse ES cells, probably due to the fact that the cell cycle of hES cells is 3 time longer than that of mouse ES cells [8-9]. Therefore, a longer time frame is required in assays to distinguish undifferentiated from differentiating or differentiated hES cells.

When an extracellular matrix mixture such as Matrigel is provided, undifferentiated hES cells can be propagated by supplementing conditioned medium (CM) from $\mathrm{pMEFs}$ to either serum-containing or serum-free media [9]. Under the latter condition, adding basic Fibroblast Growth Factor (bFGF) and insulin is necessary [8,9]. It becomes increasingly clear that external factors required for the growth of undifferentiated hES cells are different from those required for mouse ES cells. While the combination of LIF and BMP4 is sufficient to propagate mouse ES cells [10], LIF had little effect on hES cells and BMP4 induces $\mathrm{hES}$ cell differentiation preferentially to trophoectoderm [11-12]. Using multiple (supportive and non-supportive) feeder cells, and soluble recombinant factors (and their antagonists), we now have a better understanding of multiple external signals required for the growth of undifferentiated hES cells. One can envision that at least 3 types of external signals are required: 1) From factors such as insulin and neurotrophins that activate the PI3 kinase/AKT pathway and promote cell survival [13]; 2) From factors such as Wnt that activate c-Myc and cyclin D and stimulate cell proliferation $[14,15]$; 3) From factors such as TGF $\beta$, activin A, or BMP antagonists that prevent spontaneous differentiation or maintain undifferentiated $\mathrm{hES}$ cells during cell proliferation in culture [16-20]. A single growth factor may transmit multiple types of signals, or multiple factors can provide a specific type of signals such as one required for cell survival and proliferation. Indeed, many cells could provide the first two types of signals and support the survival and proliferation of hES cells, at least transiently. In contrast, pMEFs and selected human feeder cells provide an additional (the third type) signal to support the prolonged growth of undifferentiated hES cells [15-22]. Although multiple factors such as activin, TGF $\beta$ or BMP antagonists, and a high concentration of bFGF have been shown to promote the growth of undifferentiated hES cells [16-20], it is unclear whether they are sufficient to sustain the prolonged expansion of bona fide hES cells (vs. those that have been adapted or mutated during the selective culture process). In addition, it remains a challenge to develop culture conditions for the efficient and prolonged propagation of homogenous undifferentiated hES cells and the culturing of these cells at a clonal level.

We previously showed that recombinant Wnt3a proteins stimulate $\mathrm{hES}$ cell proliferation and affect cell fate determination [15]. In the absence of feeder cell-derived factors, hES cells cultured under a feeder-free condition survived and proliferated poorly. Adding recombinant Wnt3a stimulated hES cell proliferation but also differentiation [15]. This is consistent with the finding that $\beta$-catenin-mediated transcriptional activation in the canonical Wnt pathway was minimal in undifferentiated hESCs, but greatly upregulated upon induction of differentiation [15]. In the present study, we further extended our analysis to other Wnt ligands available to us. Wnt1, another ligand that activates canonical Wnt pathways, displayed a similar effect on hES cells as Wnt3a. However, Wnt5a which activates non-canonical Wnt pathways had little effect in this assay. To explore the possibility of promoting the proliferation of undifferentiated hES cells by activating the canonical Wnt signaling, we overexpressed Wnt3a or Wnt1 gene in immortalized human adult fibroblast (HAFi) cells that are able to replace pMEFs in supporting long-term growth of undifferentiated hES cells. Unlike pMEFs whose proliferation and ability to support hES cells decline after 3 passages, HAFi cells with or without a Wnt transgene can be propagated indefinitely. Over-expression of the Wnt3a gene significantly enhanced the ability of HAFi feeder cells to support the growth of undifferentiated colonies of 3 different human ES cell lines.

\section{Materials and Methods}

\section{Human embryonic stem ( $h E S$ ) cell cultures}

$\mathrm{The} \mathrm{H} 1$ and $\mathrm{H} 9 \mathrm{hES}$ cell lines were obtained from WiCell Research Institute (Madison, WI, www.wicell.org) at passage (p) 22 or p23 [1]. I-6 was obtained from Technion-Israel Institute of Technology at p30. We propagated hES cells on pMEFs or HAFi cell as previously described $[15,21]$. I- 6 hES cells were propagated by collagenase IV following the provider's instruction. Undifferentiated H1 hES cells from p35-80, H9 cells of p29-73, and I-6 of p30-64 with a normal karyotype were used in this study. Approximately once every 2-3 months, hES cells were karyotyped and their ability to form teratomas in SCID-beige mice was also tested [15, 23].

\section{Immortalized HAFi and derivatives as feeder cells}

HAFi cells derived from human skin fibroblasts and hTERT transduction have been previously described $[15,23]$. They triplicate every week for years, and maintain a typical and non-transformed fibroblast phenotype. For use as feeders, HAFi cells or derivatives were irradiated ( $40 \mathrm{~Gy}$ ) and plated at a density of $\sim 2.5 \times 10^{4}$ cells $/ \mathrm{cm}^{2}$ $\left(5 \times 10^{4}\right.$ cells per well in 24 well plates). Although hES cells grow slightly slower in the first passage after switching from pMEF to HAFi feeder cells, the growth of hES cells in subsequent passages were at least as good as on the pMEFs feeders that we have used. The co-culture with hES cells were replenished daily with the hES cell medium. After 3-9 d, the co-cultures were either passaged along after split or stained for AP activity. All the feeder cells were negative for AP, while undifferentiated hES cell colonies stained densely for AP $[15,23]$.

Immortalized HAFi cells can be transfected by a plasmid, or stably transduced by a retroviral or lentiviral vector more efficiently. To make stable HAFi derivatives over-expressing Wnt1 or Wnt3a 
proteins (tagged with the HA epitope), we used two existing retroviral vectors as a gift from Dr Hongjun Song (Johns Hopkins University, JHU). The retroviral vectors (LTR-neo-CMV promoter-Wnt1 or Wnt3a gene with the HA tag) were produced in $293 \mathrm{~T}$ cells by transient transfection as previously described [24]. Stably transduced cells were selected by G418 $(500 \mu \mathrm{g} / \mathrm{ml})$. In addition, we have also created puromycin-resistant HAFi cells (HAFi-puro) with a retroviral vector, and Wnt3a-producing HAFi cells by a lentiviral vector. In both cases, the puromycin resistance gene is controlled by the PGK promoter.

Western blot to monitor the over-expression of Wnt proteins in HAFi cells

HAFi cells with or without the stably-transduced Wnt gene were harvested and analyzed by Western blotting for the production of recombinant Wnt protein that is tagged by the hemaggutinin (HA) epitope. The blot was first probed by the anti-HA rabbit antibody (1: 500 or $2 \mu \mathrm{g} / \mathrm{ml}$ ) purchased from Abcam, UK. After stripping, the blot was re-probed by a mouse monoclonal antibody recognizing the $\beta$-actin (clone AC-15, 1:5 000, from Sigma, USA).

\section{A luciferase reporter assay to measure the Wnt mediated transcriptional activation}

The Wnt activity that activates its canonical ( $\beta$-catenin mediated) signaling pathway was assayed using the STF reporter cells that stably express a luciferase reporter gene driven by the TCF optimal promoter (TOP) [25]. The STF is derived from 293 cells containing the TOP-luciferase reporter vector. $4000 \mathrm{STF}$ cells were co-cultured overnight with $1.6 \times 10^{4} \mathrm{HAFi}$, HAFi cells transduced with Wnt1 or Wnt3a, or 293T (as a negative control). Cells were then harvested and lysed with Glo lysis buffer (Promega, Madison, USA) and the luciferase activity was measured as previously described [15].

\section{The feeder-free culture of hES cells}

We often used the feeder-free culture system described previously with modifications $[9,23]$ to reduce the numbers of carryover feeder cells and perform growth factor requirement assays. Matrige ${ }^{\mathrm{TM}}(\mathrm{BD}$ Biosciences, Bedford, MA, USA) was diluted at 1:30 to coat the plate and used as extracellular substrates. Human ES cells were cultured on Matrigel with the pMEF conditioned medium (CM), which provides soluble factors required for cell survival/proliferation and maintenance of undifferentiated hES cells. Recombinant Wnt3a and Wnt5a proteins were purchased from R \& D Systems (Minneapolis, MN, USA). Wnt1 was purchased from Peprotech, USA. As expected, Wnt 1 and Wnt3a, but not Wnt5a, activate the canonical Wnt signaling pathway and a TOP luciferase reporter.

To test the effects of growth factors such as Wnt1, hES cells were cultured on Matrigel with pMEF-CM for at least one passage to reduce the numbers of carryover pMEFs from previous co-cultures as described [15]. After harvest by $0.05 \%$ trypsin $/ 0.5 \mathrm{mM}$ EDTA, hES cells in small clumps ( $\sim 10-20$ cells $)$ were seeded on Matrigel-coated 24 -well plates $\left(1.5 \times 10^{4}\right.$ cells per well $)$ with pMEF-CM or the plain $\mathrm{hES}$ cell medium, in the presence or absence of Wnt $(100 \mathrm{ng} / \mathrm{ml}$ unless otherwise indicated). The culture medium was changed daily. At day 4-5 when largest colonies in the positive control reached confluency, the cells in each group were harvested and counted using $0.4 \%$ trypan blue solution. A fraction of harvested cells were also seeded on pMEF feeder cells to assess numbers of hES cell capable of forming colonies subsequently. The undifferentiated hES cell colonies formed on pMEFs were counted after AP staining. The $\mathrm{AP}^{+}$colony-forming units are used to quantify numbers of undifferentiated hES cells in the test population [23].

\section{Stable transfection of human ES cells by a plasmid vector}

To assess transfection of hES cells, we used both enhanced green fluorescent protein (GFP) and an improved red fluorescent protein (RFP) called tandem dimer (td) Tomato $(E x=554 \mathrm{~nm}, \mathrm{Em}=581 \mathrm{~nm})$ as reported recently [26]. A cDNA3.1-based plasmid expressing the tdTomato RFP reporter was provided by Dr. Nicolas Christoforou (JHU). The plasmid also contains a neomycin resistance gene controlled by the SV40 promoter. The circular plasmid $(5 \mu \mathrm{g})$ was transfected into $\sim 2 \times 10^{6} \mathrm{hES}$ cells by Amaxa's electroporation method ("nucleofection ${ }^{\mathrm{TM}}$ ) using the mouse ES cell solution and Program A23 of Nucleofector II, following the manufacturer's recommendation. Treated cells were plated into 3 wells of HAFi/Wnt3a feeder cells (in a 6-well plate, after 40 Gy irradiation). Two days after, 100 $\mu \mathrm{g} / \mathrm{ml} \mathrm{G} 418$ were added. Transfected cells were passaged onto fresh $\mathrm{HAFi} / \mathrm{Wnt} 3 \mathrm{a}$ cells as needed. Similar results were obtained by using lipofectamine 2000-mediated DNA transfection (Invitrogen).

\section{Results}

\section{Recombinant Wnt1 but not Wnt5a acted similarly as Wnt 3 a on $h E S$ cells}

In addition to Wnt3a, we also tested the effect of recombinant Wnt1 and Wnt5a proteins on hES cells. As described in our previous study with Wnt3a [15], hES cells were cultured on Matrigel in the presence or absence of $\mathrm{CM}$ from pMEFs. When CM was absent, an equal volume of the hES cell medium (containing insulin and bFGF) was added. Wnt1 or Wnt5a were added in the presence or absence of CM. In the absence of pMEF-CM, a few cell survived after $5 \mathrm{~d}$ in culture (Figure 1A). The addition of Wnt1 into the hES cell medium provided a cell survival/ proliferation advantage similar to what was observed previously with Wnt3a [15]. The promotion of Wnt1 on hES cell growth is also obvious in the presence of CM (Figure 1A). The harvested cells were further tested for the content of undifferentiated hES cells based on their ability to form undifferentiated colonies. Numbers of $\mathrm{AP}^{+}$, compact hES colonies (from 1000 cells plated onto pMEFs) were counted and shown in Figure 1B. Cells cultured with Wnt1 in the hES cell basal medium could not maintain the undifferentiated (self-renewing) state of hES cells. In contrast, addition of Wnt1 into CM doubled the total cell number and did not alter the frequency of undifferentiated (colony-forming) hES cells (Figure 1B). While the Wnt1 effect is very similar to Wnt3a as we previously described [15], Wnt5a (up to $100 \mathrm{ng} / \mathrm{ml}$ ) showed little effect in this system (data not shown). Combined with other evidence, these data indicated that Wnt ligands such as Wnt1 and Wnt3a that activate the canonical Wnt signaling pathway can stimulate proliferation of hES cells. In the absence of $\mathrm{CM}$, the stimulation of hES cell proliferation by Wnt1 and Wnt3a is also accompanied with acceleration of differen- 

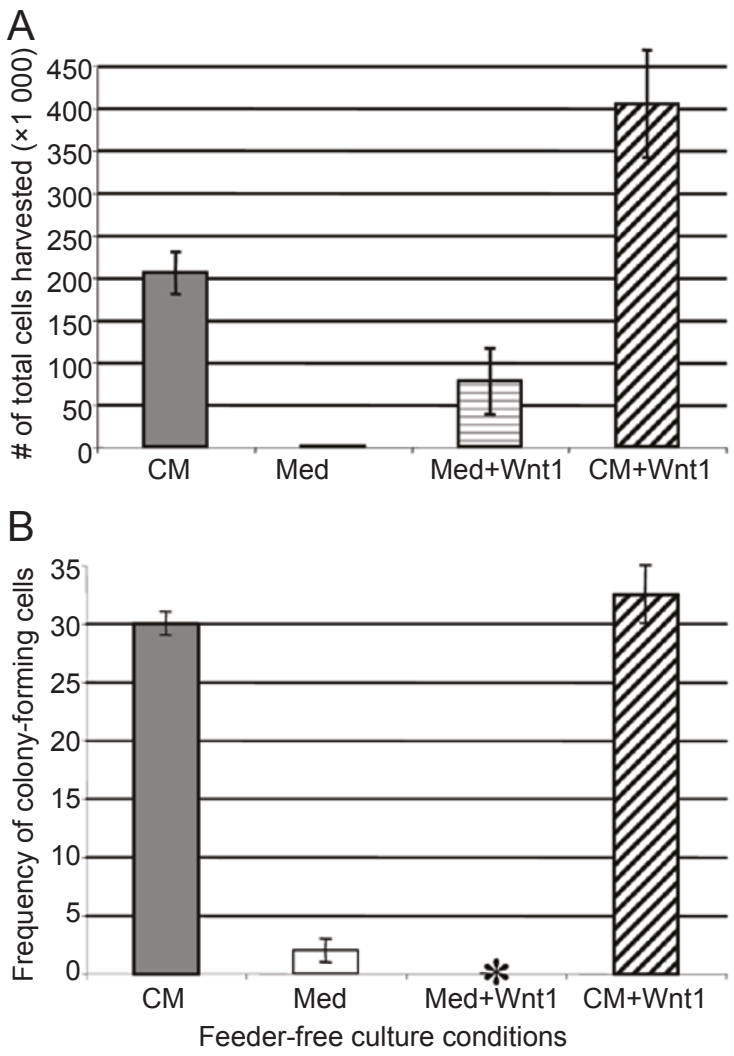

Figure 1 Wnt 1 stimulates the growth of hES cells in the presence or absence of CM from primary mouse embryonic fibroblasts (pMEFs). H1 hES cells were cultured in 24-well plates (15 000 cells/well) coated with Matrigel in the presence or absence of CM. When CM was absent, an equal volume of the hES cell medium (Med) containing insulin and bFGF was added. Wnt1 $(20 \mathrm{ng} / \mathrm{ml})$ was added in the presence or absence of CM, and the media were changed daily. After 5 days, total cells were harvested and numerated. Mean and $\mathrm{SD}(\mathrm{n}=2)$ were plotted in (A). To assess undifferentiated hES cells, 1000 cells of each population were seeded on the pMEFs to allow the growth of undifferentiated colonies. After 6 days, alkaline phosphatase positive $\left(\mathrm{AP}^{+}\right)$colonies were numerated after AP staining, as an index of undifferentiated hES cells. Mean $\pm \mathrm{SD}$ of $\mathrm{AP}^{+}$colonies per 1000 input cells $(n=3)$ were plotted in $(\mathbf{B}) . * 3000$ cells cultured with hES medium and Wnt 1 for 5 days did not give rise to a single $\mathrm{AP}^{+} \mathrm{hES}$ cell colony.

tiation and loss of self-renewing capacity. However, in the presence of CM that contains renewing factors, Wnt1 (and Wnt3a) enhanced the growth of undifferentiated hES cells that are able to generate colonies of undifferentiated hES cells (i.e., self-renewal).

Wnt accelerates hES cell proliferation and differentiation when $b F G F$ is absent

We also used a second strategy to assess the role of the Wnt canonical signaling pathway. It has been previously shown that, when the serum-free medium is used, exogenous bFGF is required for the renewing proliferation of $\mathrm{hES}$ cells. This is also true under the feeder-free culture condition whereby $\mathrm{hES}$ cells are cultured on Matrigel together with $\mathrm{pMEF} C \mathrm{CM}$ that provides other growth factors required for $h E S$ cell survival and proliferation $[8,9]$. Thus, we made pMEF CM in the presence or absence exogenous bFGF. Human ES cells were cultured as before with either type of $\mathrm{CM}$ (-bFGF and $+\mathrm{bFGF})$, and in the presence or absence of Wnt3a (Figure 2A). When the-bFGF CM was used, hES cells survived and passaged well on Matrigel at least for $15 \mathrm{~d}$ (vs 4-5 d when CM was absent, Figure 1A), similar to the case when the $+\mathrm{bFGF} C \mathrm{CM}$ was used. Adding Wnt3a led to a moderate increase of cell numbers with both types of CM (-FGF and $+\mathrm{bFGF})$. The resulting cells were then analyzed, based on the presence of undifferentiated markers such as SSEA-3 (Figure 2B and 2C) and the ability to form undifferentiated hES colonies (Figure 2D). Adding Wnt3a did not alter the high level expression of SSEA-3 when the + bFGF CM was used (Figure 2B). Under the culture condition with -bFGF CM, however, adding Wnt3a resulted in the loss of SSEA-3 expression (Figure 2C), indicating that the treated cells were differentiated. The harvested cells were further tested in a hES cell colony formation assay as in Figure 1B. When the -bFGF CM was used for $15 \mathrm{~d}$, undifferentiated hES cells (capable of forming colonies in the subsequent assay) were still abundant, but diminished when Wnt3a was added (Figure 2D). In contrast, adding Wnt3a to the +bFGF CM moderately increased the frequency of colony forming undifferentiated hES cells as well as the total cell number as seen in Figure 2A. These data better illustrated the Wnt "activation" hypothesis that we have previously proposed [15]. In the absence of a sufficient level of bFGF or CM-derived factors that are required to maintain hES cells at an undifferentiated state, the canonical Wnt signaling activation accelerates cell proliferation as well as differentiation. In the presence of bFGF and supportive feeder cells (or CM), however, Wnt will stimulate the self-renewing proliferation of the undifferentiated hES cell population.

\section{Modulating Wnt activity using Wnt-overexpressing feeder cells}

Although several recombinant Wnt proteins became available recently, it remains technically challenging to purify bioactive Wnt proteins. They are lipid-modified, tightly associated with extracellular matrix of Wnt-producing mammalian cells, and poorly released to culture media naturally $[27,28]$. One way to overcome the limitation is to directly use Wnt over-expressing cell lines. To propagate hES cells more efficiently, we decided to over-express Wnt1 or Wnt3a in the immortalized human adult fibroblasts 
A Numbers of harversted cells $\left(10^{3} /\right.$ well)

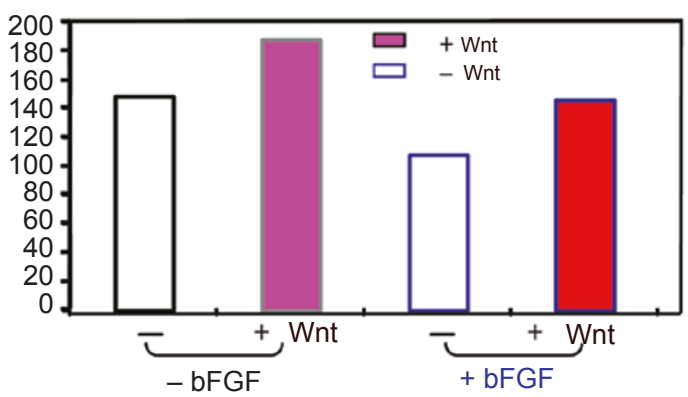

D Undiff. hES cell colonies $/ 10^{4}$ cells

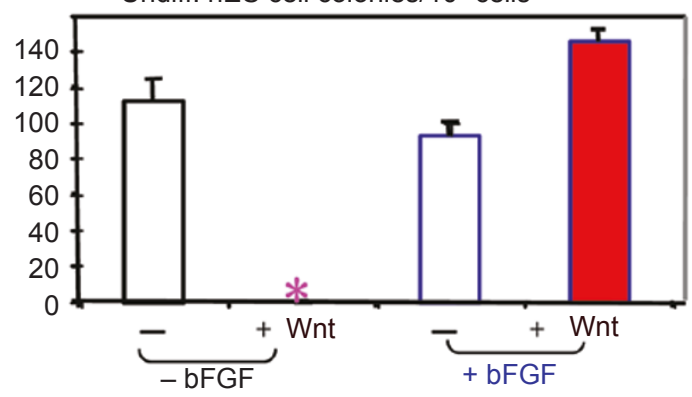

B SSEA-3 staining for cells + bFGF

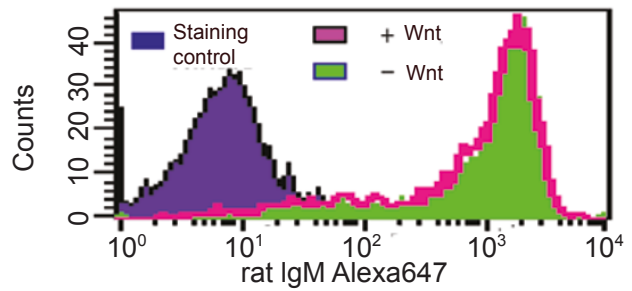

C

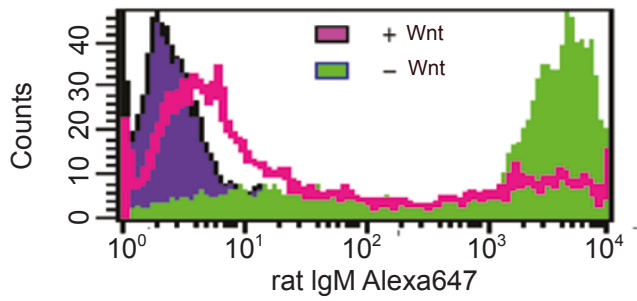

Figure 2 Wnt stimulates human ES cell proliferation, but also differentiation when exogenous bFGF is absent. H1 ES cells were cultured on Matrigel with two types of the CM from pMEFs. In addition to the standard CM which bFGF (4 ng/ml) was added before and after CM collection ( $+\mathrm{bFGF}$ ), we also made CM devoid of exogenous bFGF (-bFGF). The -bFGF CM supported the growth of hES cells for at least 15 days ( 3 consecutive passages), in the presence or absence of Wnt3a (100 ng/ml). (A) Numbers of harvested cells after 15 days \pm Wnt3a and in the presence of - bFGF and + bFGF CM. Aliquots of cells were analyzed for the expression of an undifferentiated marker SSEA-3 (B and C). Flow cytometric analysis of cells cultured with the $+b F G F$ and -bFGF CM, with or without Wnt3a. The blue lines were the background staining from an irrelevant control antibody. While hES cells treated with (red) or without (green) Wnt3a showed a similar level of high SSEA-3 expression after cultured with +bFGF CM (B), the Wnt3a-treated cells (red) lost the SSEA-3 expression greatly after culturing with the -FGF CM (C). (D) Colony forming assays for the level of undifferentiated hES cells in each population in (A). 10000 cells from each population were plated on pMEF feeder cells under the optimal culture condition (+bFGF) as in Figure 1B. Six days after, undifferentiated hES cell colonies were stained for alkaline phosphatase and counted. Mean \pm SD $(n=3)$ was plotted. The cells cultured with $-b F G F C M$ in the presence of Wnt3a did not form any undifferentiated compact hES colonies $(*$, from 30000 cells), while the three other samples showed comparable frequencies of undifferentiated hES cell colonies.

(HAFi) cells we established previously [15, 23]. As shown previously, H1 or I-6 hES cells maintained undifferentiated morphology even after 6 consecutive passages on the parental HAFi feeder cells or a derivative expressing the puromycin selection gene, HAFi-Puro (Figure $3 \mathrm{~A}$ and $3 \mathrm{~B}$ ). Human ES cells cultured on HAFi had a similar or slightly better growth rate than that on pMEFs, formed embryoid bodies in vitro and teratomas in vivo [23].

After stable transduction by a retroviral vector expressing HA-tagged Wnt1 or Wnt3a (and co-expressing the neomycin resistance gene), selected HAFi cells (HAFi/ Wnt1 or HAFi/Wnt3a) maintained a similar growth rate (triplication per week, data not shown). We also examined the relative level of HA-tagged Wnt1 or Wnt3a proteins produced by transduced HAFi cells by Western blot using the HA-specific antibody (Figure 3C). As expected, the HA antibody detected a protein band of predicted sizes (42 KDa for Wnt1-HA and $40 \mathrm{KDa}$ for Wnt3a-HA) in $\mathrm{HAFi} / \mathrm{Wnt} 1$ and HAFi/Wnt3a cells, respectively, but not in the parental HAFi cells. The level of Wnt3a proteins is about 3 fold higher than that of Wnt1. By the same assay, however, we have not been able to detect HA-tagged soluble Wnt proteins released in the conditioned medium from either HAFi/Wnt1 or HAFi/Wnt3a cells. Similarly, we did not find a detectable level of soluble activity that activates the Wnt canonical pathway in a responding cell line STF expressing a TOP-luciferase reporter [25]. This is consistent with the notion that Wnt proteins that are lipid-modified and tightly associated with the extracellular matrix are poorly released to culture media [27]. 
To detect the cell- or extracellular matrix-associated Wnt activity, we cultured Wnt producing HAFi cells together with the STF reporter cells that express a TOP-luciferase reporter [25]. The parental HAFi cells showed little activity as compared to the negative control 239T cells as expected (Figure 3D). The HAFi/Wnt1 cells showed some while HAFi/Wnt3a cells showed the best activity in this surrogate assay, consistent with the fact that more Wnt3a proteins are expressed in HAFi/Wnt3a cells than Wnt 1 in HAFi/Wnt1 cells. In a preliminary test with $\mathrm{H} 9$ hES cells, the HAFi/Wnt3a cells supported the growth of undifferentiated $\mathrm{hES}$ cells measured as $\mathrm{AP}^{+}$colonies after two passages (Figure 3E).
Wnt-producing HAFi feeder cells are more efficient to support hES cells

We next examined whether these Wnt-expressing HAFi cells are more potent to support the growth of undifferentiated hES cells, including different hES cell lines that grow poorly at a clonal level. For example, we had to propagate the I-6 hES cell stock by partially dissociating hES cell colonies using collagenase and passaging them as fairly large cell clumps. The expansion efficiency of these cells was poor and it is very difficult to achieve clonal expansion from single cells either on pMEFs or HAFi feeder cells. With Wnt-producing HAFi cells, we examined whether clonal growth of I-6 hES cells would be more efficient
A

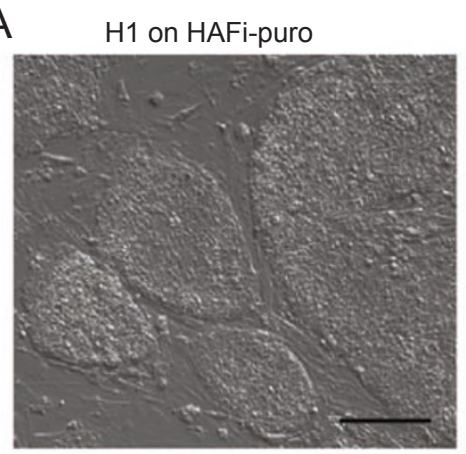

B

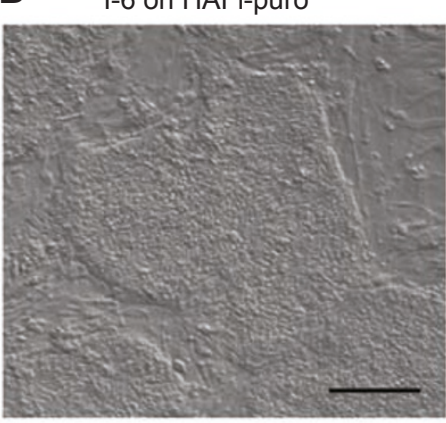

C

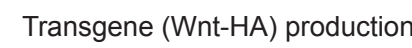
in transduced HAFi cells

HA blot

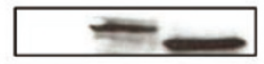

$\beta$-Actin blot

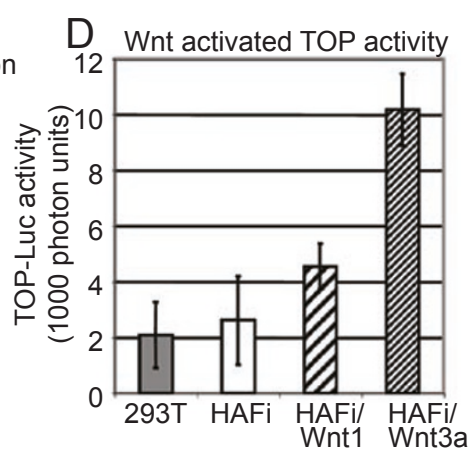

E

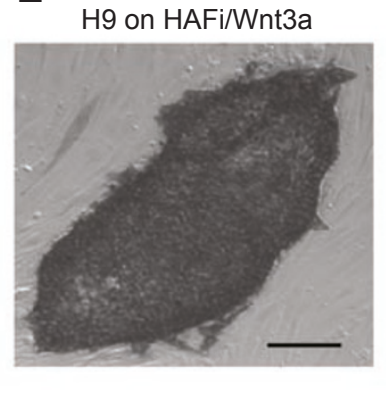

Figure 3 Immortalized HAFi feeder cells and their derivatives over-expressing Wnt. In addition to the original HAFi cells, we have also made the derivative expressing the puromycin (HAFi-Puro) resistance gene (A and $\mathbf{B}$ ), and those expressing Wnt1 or Wnt3a (C-E). Bar scale: $100 \mu \mathrm{m}$ in (A, B and E). H1 hES cells (A) or I-6 cells (B) were passaged as small clumps as usual on HAFi-puro feeder cells. Undifferentiated ES cell clones were evident after 6 passages of continuous culture on HAFi-puro as on HAFi feeder cells. (C-E) HAFi cells stably expressing Wnt together with the neomycin resistance gene after retroviral transduction. (C) After transduction with vectors expressing Wnt1 (lane 2) or Wnt3a (lane 3), expanded HAFi/wnt1 and HAFi/Wnt3a cells were analyzed for production of Wnt proteins (tagged with HA epitope), together with parental HAFi cells (lane 1). Lysates from 3 HAFi cell types were made and analyzed by western blot using specific antibodies recognizing either the HA epitope (fused with the Wnt protein) or beta-actin (as a loading control). The HA antibody detected HA-tagged proteins in HAFi/Wnt1 cells ( 42 kDa) and HAFi/Wnt3a cells $(\sim 40 \mathrm{kDa})$ as expected. After striping, the blot was re-probed by a beta-actin antibody, which detected a constitutive protein of $42 \mathrm{kDa}$ in all 3 cell types as expected. (D) The production of Wnt activation activity by HAFi cells was also measured by co-culture with a Wnt-responsive reporter cell line STF expressing the TOP-luc reporter. The mean \pm SD ( $n=3$ ) of the Wnt/TOP activated luciferase activity (in 1000 photon units) by each type of HAFi cells and a negative control (293T cells) were plotted. (E) H9 cells were cultured on Wnt3a-producing HAFi cells (HAFi/Wnt3a) for two passages and then stained for the alkaline phosphatase marker. See Figure 4 for more quantitative data. 

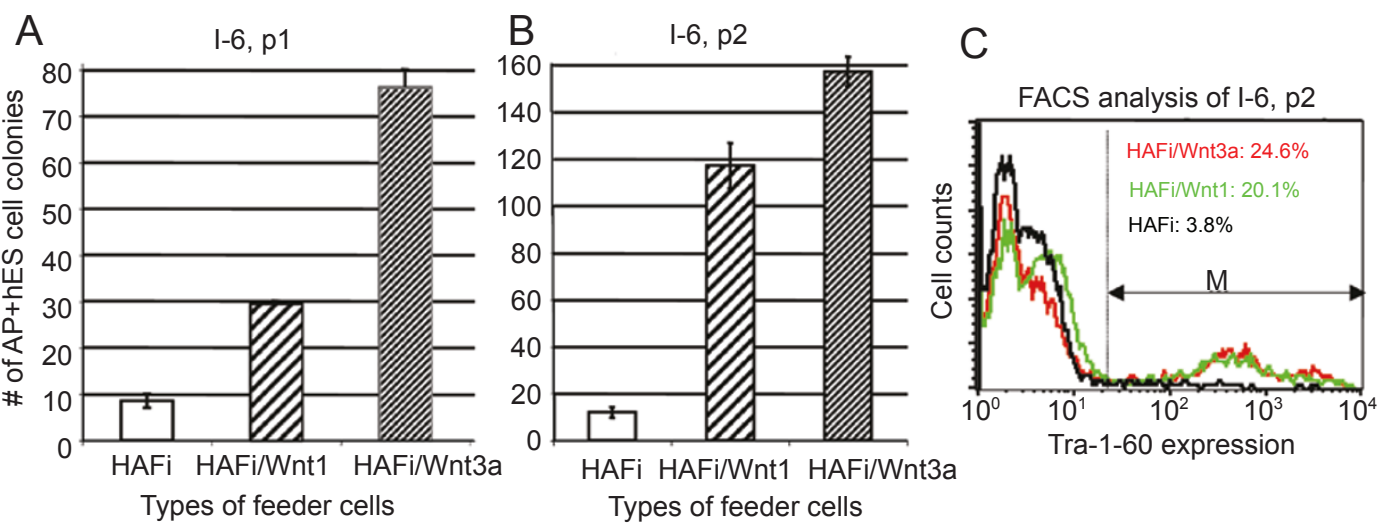

D
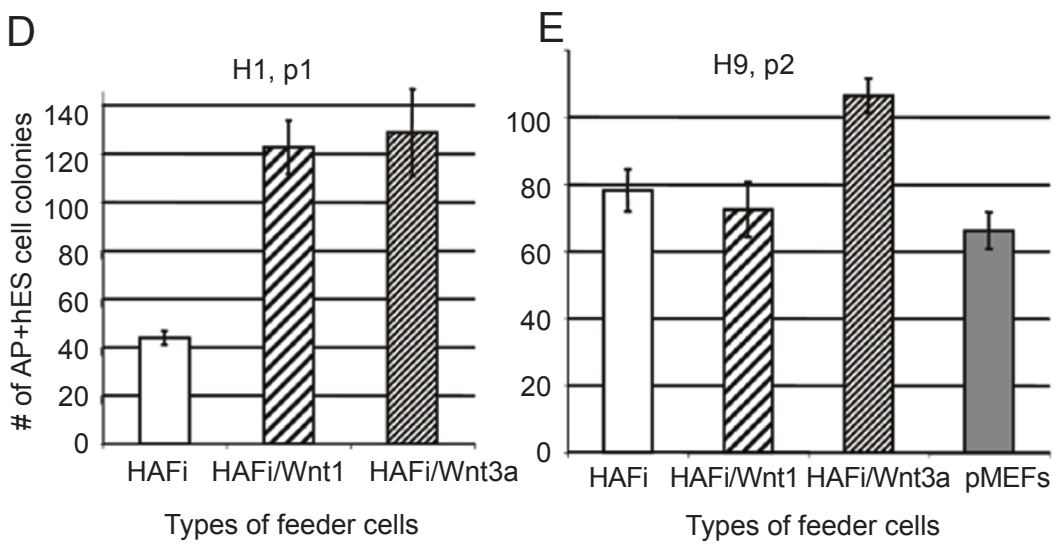

Figure 4 Wnt-producing HAFi cells better support the undifferentiated growth of 3 hES cell lines. (A-C). I-6 hES cells after one (A) or two passages (B-C) on various versions of HAFi cells. I-6 hES cells (p64) that were maintained on pMEFs and passaged by collagenase IV were plated on Matrigel for one passage. Then hES cells were digested by Trypsin/EDTA to achieve single cell suspension. 5000 cells were plated in each well in 24-well plates $(n=4)$ that were coated with irradiated HAFi feeder cells. After $9 \mathrm{~d}$, a half of wells were stained for $\mathrm{AP}^{+}$colonies $\mathbf{( A )}$ and the second half were harvested and re-plated (1:3 split) as before. After additional $5 \mathrm{~d}$, one half of wells were stained $(n=3, \mathbf{B})$ and the second half were harvested for FACS analysis. The mean \pm SD of AP colonies after the first and second passage were plotted. (C) FACS of I-6 cells after co-culture with HAFi cells for 14 d. Harvested cells were stained for an undifferentiated hES surface marker Tra-1-60 and the histograms were overlayed. hES cells co-cultured with HAFi (black), HAFi/Wnt1 (green) and HAFi/Wnt3a (red) were identified in the marked (M) region. The percentage of Tra-1-60 positive (undifferentiated hES) cells in each co-culture is also denoted. HAFi cells ( \pm Wnt) are Tra-1-60 negative. (D) Similarly, 5,000 H1 hES cells were assayed for one passage $(6 \mathrm{~d}, \mathrm{n}=4)$. (E) H9 (p73) hES cells were assayed on 3 types of HAFi cells and pMEFs. 3 000 cells per well were seeded in the first passage and re-plated after 1:4 split. After a total of $9 \mathrm{~d}$ in co-culture, $\mathrm{AP}^{+}$colonies were counted. Mean $\pm \operatorname{SD}(n=4)$ with 4 types of feeder cells were plotted as before. Note that the colony formation efficiency and growth rate of the H9 hES cells were much higher than the I-6 and H1 hES cells.

(Figure 4A and 4B). Single cell suspension was plated on the 3 types of HAFi cells. At the end of first passage, 50\% of the wells were stained for $\mathrm{AP}^{+}$colonies (Figure 4A) and the other $50 \%$ were passaged (after 1:3 split) onto the same type of HAFi feeder cells followed by the count of $\mathrm{AP}^{+}$ colonies (Figure 4B). The Wnt3a-producing HAFi cells were 7-8 fold more efficient than the parental HAFi cells after the first passage and $>13$ fold after the second passage in supporting the clonal growth of undifferentiated hES cells (Figure 4B). The I-6 cells after 2 passages ( $14 \mathrm{~d}$ ) were also analyzed by FACS analysis for the expression of an undifferentiated marker Tra-1-60 (Figure 4C). The Tra-1-60 expression after $14 \mathrm{~d}$ remained high after co-culture with the 3 types of HAFi cells. Co-culture with Wnt3a-producing HAFi cells resulted in the most numbers of Tra-1-60 cells (24.6\%), while Tra-1-60 negative cells were likely HAFi or derivative cells harvested from co-cultures (Figure 4C). Similar data were obtained with later passage H1 (Figure 4D) and H9 (Figure 4E) hES cells that grew more efficiently, although the difference was less than that observed with I-6 cells. Nonetheless, Wnt3a-producing HAFi cells (in short WHAFi) cells gave the best results for 3 differ- 

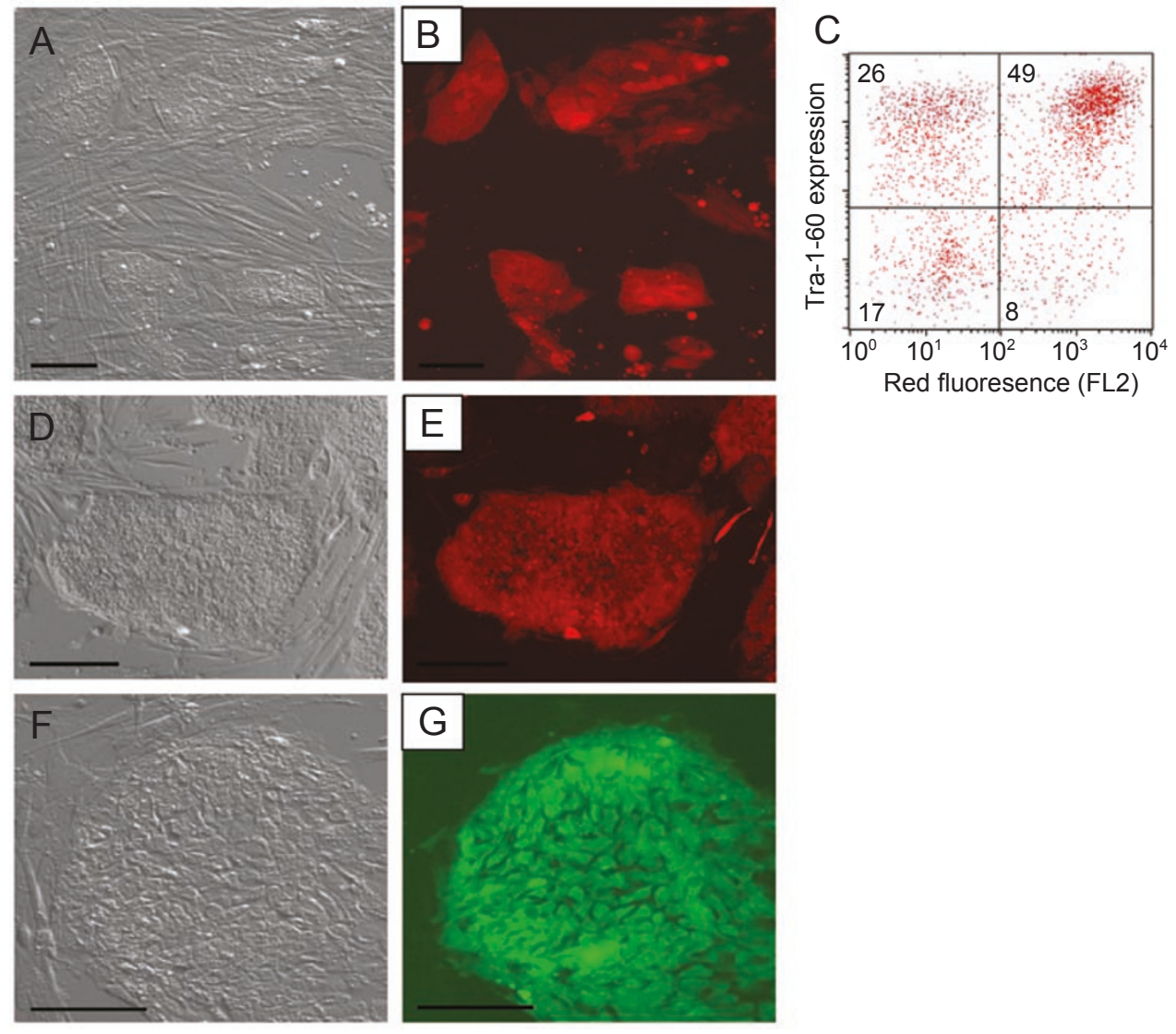

Figure 5 Wnt3a-producing HAFi cells support the growth of transfected or transduced hES cells stably expressing RFP and GFP reporter genes (A and B) H9 hES cells were transfected by a plasmid co-expressing the neomycin resistance gene and an improved red fluorescent protein (RFP) called tdTomato. Human ES cells were then plated on Wnt3a-producing HAFi (WAHFi) feeder cells (that are G418 resistant) that had been irradiated and plated in 6-well plates one or a few days before. After 3 passages, G418-resistant stably transfected colonies emerged, and further expanded. Micrographs of colonies expressing the tdTomato RFP were taken using either bright field (A) or a fluorescence filter suitable for the RFP (B). After prolonged cell expansion ( $>5$ passages), the stable transgene expression (tdTomato RFP) in undifferentiated hES cells (Tra-1-60 positive) was examined by flow cytometry (C). The percentage of each cell population is denoted in each quadrant. The majority of undifferentiated hES cells is RFP+ and the vast majority of RFP+ cells expressed the Tra-1-60 undifferentiated marker. WHAFi cells were further engineered to be puromycin-resistant. The new feeder cells W3R are resistant to the selection of 3 antibiotics. (D and E). Micrographs of co-cultures of W3R and H9 cell colonies stably expressing the monomeric cherry RFP after transduction by a vector co-expressing the cherry RFP gene and the puromycin-resistance gene, and after puromycin selection and expansion. (F and $\mathbf{G})$. Similarly, W3R cells support the growth of $\mathrm{H} 1 \mathrm{hES}$ cells that are stably transduced by GFP. Bar scale: $100 \mu \mathrm{m}$.

ent hES cell lines, and were thus used more frequently in subsequent experiments.

Creation and selection of rare stably-transfected hES cell clones after plasmid transfection

It has been difficult to obtain stably-transfected hES clones after plasmid transfection, despite that the transient transfection efficiency is as high as $40-60 \%$ by both lipofectamine 2000 and eletroproation (Amaxa) methods. This is likely due to at least in part the poor growth of hES cells at a clonal level $(\leq 1-2 \%)$ as compared to mouse ES cells (10-30\%). With the WHAFi feeder cells that are also neomycin (G418) resistant, we tested if we could obtain stable clones of hES cells after transfection by an existing plasmid co-expressing the improved red fluorescent protein (RFP) called tdTomato [26]. After sustained G418 selection of transfected hES cells cultured on WHAFi feeder cells, many colonies emerged. When the plasmid co-expressing tdTomato RFP was used, $\sim 20 \%$ of stable H9 hES cell colonies expressed bright red fluorescence as shown in Figure 5A and 5B. After G418 selection on WHAFi cells, many undifferentiated colonies remained RFP posi- 
tive but RFP negative colonies were also observed. After extensive passages and expansion, hES cells cultured on WHAFi feeder cells were harvested and analyzed by flow cytometry (Figure 5C). Among undifferentiated hES cells (Tra-1-60 positive), the majority expressed a high level of red fluorescence from the tdTomato RFP reporter. We also made a derivative of WHAFi cells by stable transduction with a vector expressing the puromycin resistant gene. This WHAFi derivative is dubbed as W3R, because of its resistance to the selection by 3 commonly-used antibiotics: hygromycin, neomycin and puromycin. Micrographs (Figure 5D and 5E) show $\mathrm{H} 9$ cell colonies stably expressing the monomeric cherry (mCherry) RFP gene after transduction by a vector co-expressing the mCherry RFP gene and the puromycin-resistance gene. Similarly, W3R feeder cells also support the growth of $\mathrm{H} 1 \mathrm{hES}$ cells that were stably transduced by a GFP vector (Figure $5 \mathrm{~F}$ and $5 \mathrm{G}$ ). These experiments demonstrated the utility of WHAFi or W3R cells to support the growth of clonal hES cells.

\section{Discussion}

Pleiotrophic Wnt cytokine family members play important roles in many developmental events [29-31]. It is now clear that there are at least two types of Wnt signaling pathways: one is dependent on the $\beta$-catenin mediated activation that turns on the expression of a group of genes via TCF/LEF nuclear factors and the second is $\beta$-catenin independent. The latter is less defined and commonly referred as the non-canonical Wnt signaling pathway [32]. The Wnt canonical signaling pathway is known to be associated with cell proliferation during the embryonic development, tissue regeneration after injury and tumor formation, among many other functions $[29,30]$. In recent years, the Wnt canonical signaling is also implicated to support stem cell selfrenewal or differentiation, although the exact role of the Wnt canonical signaling was unclear until recently $[27,28$, 31-43]. On one hand, Wnt appears to be an important factor for stem cell self-renewing that requires cell division. For example, in a widely cited study, Wnt/ $\beta$-catenin activation appeared to expand murine hematopoietic stem/progenitor cells in vitro when other signals were also present $[27,28]$. In vivo, Wnt/ $\beta$-catenin activation is commonly observed in the "activated" stem cells as well as subsequently derived (transiently-amplifying) progenitor cell populations [3840]. On the other hand, Wnt activation is also found to be required for cell specification (i.e., differentiation) from a more primitive stem cell precursor [35-37]. Thus, the Wnt canonical signaling does not appear to act on stem cell maintenance that prevents differentiation from stem cells. These data are contradictory to the "maintenance" model that was proposed previously to explain the roles of Wnt on stem cells and their self-renewal.

In contrast, the "activation" model proposed recently is also better in explaining the diverse or even contradictory roles of Wnt signaling in regulating stem cells [15, 40]. In this "activation" model, Wnt canonical signaling stimulates stem cell activation, survival or proliferation, which is required for either self-renewal or differentiation (that generates progeny cells). Thus the Wnt activation can enhance "stem cell fate determination": whether the term is used to describe the expansion of undifferentiated stem cells, or cellular differentiation ("specification"). In adult stem cell systems, stem cell self-renewal and differentiation does not have to be mutually exclusive, e.g., in embryonic development or a setting after tissue injury. The regenerative process requires the transient activation of resting stem cells by Wnt and other proliferative signals, generation and expansion of committed progenitor cells, and finally replacement of terminal differentiated cells that perform specified functions. The "activation" model for the role of $\mathrm{Wnt} / \beta$-catenin activation in regulating adult stem cells is further supported by the results in two recent papers, which are contradictory to the prediction from the "maintenance" model [41-43].

The data presented here provide additional evidence to the "activation" model in which the Wnt signaling serves as an "amplifier or accelerator" for hES cells, analogous to what is observed with adult stem cells. In the absence of sufficient levels of factors such as bFGF or CM-derived factors that are required to sustain an undifferentiated state, the Wnt activation accelerates cell proliferation as well as differentiation. In the presence of bFGF and supportive feeder cells (or $\mathrm{CM}$ ), however, Wnt stimulates the self-renewing proliferation of the undifferentiated hES cell population. Based on the "activation" model, we over-expressed Wnt1 or Wnt3a in immortalized adult human fibroblasts (HAFi) to enhance the expansion of undifferentiated hES cells. Testing with $3 \mathrm{hES}$ cell lines showed that Wnt3a-overepression in HAFi feeder cells further enhanced cell proliferation as well as colony formation of undifferentiated hES cells. With the features of unlimited (immortal) growth and the convenience of using one or more drug selection, Wnt3aproducing HAFi cells such as WHAFi or W3R cells will improve our ability to grow either wildtype or geneticallymodified hES cells co-expressing one or all 3 commonly used drug-resistance genes. It is also conceivable that immortal WHAFi or W3R cells can be further improved by expressing other genes encoding growth-promoting factors once they are identified. The ability of WHAFi or W3R cells to support the derivation of hES cell lines from blastocysts is unknown, but it is tempting to speculate that they could be more efficient than pMEF or primary human fibroblasts that often vary from batch to batch. 
Although the current study focused on the initial cell fate determination of undifferentiated hES cells, modulating Wnt signaling is also likely useful to promote differentiation as observed in the absence of bFGF (Figure 2). Depending on other extrinsic signals and cell-intrinsic factors, Wnt activation using feeder cells or soluble factors could also help directed differentiation from $\mathrm{hES}$ cells to generate (and expand) committed progenitors cells of specific lineages.

\section{Acknowledgment}

We thank Drs Qian Xu and Jeremy Nathans (JHU) for providing STF reporter cells, Dr Hongjun Song (JHU) for retroviral vectors over-expressing Wnt1 and Wnt3a genes. We also thank Dr Nicolas Christoforou for providing the tdTomato expression vector, and Drs Christoforou, Peter Donovan and Ms. Kristi Hohenstein for sharing experience with Amaza's nucleofection of human ES cells. This work was supported in part by research grants from National Institutes of Health (HL73781), Stem Cell Research Foundation (S2005-026), and the Johns Hopkins Institute for Cell Engineering. L Cai and C Zhou are funded in part by the Ministry of Health (2004-468) in China. L Cheng is a recipient of an International Collaboration Award (\#30428010) from the National Natural Sciences Foundation of China.

\section{References}

1 Thomson JA, Itskovitz-Eldor J, Shapiro SS, et al. Embryonic stem cell lines derived from human blastocysts. Science 1998; 282:1145-1147.

2 Reubinoff BE, Pera MF, Fong C, et al. Embryonic stem cell lines from human blastocysts: somatic differentiation in vitro. Nat Biotechnol 2000; 18:399-404.

3 Richards M, Fong CY, Chan WK, et al. Human feeders support prolonged undifferentiated growth of human inner cell masses and embryonic stem cells. Nat Biotechnol 2002; 20:933-936.

4 Cheng L, Hammond H, Ye Z, et al. Human Adult Marrow Cells Support Prolonged Expansion of Human Embryonic Stem Cells in Culture. Stem Cells 2003; 21:131-142.

5 Amit M, Margulets V, Segev H, et al. Human feeder layers for human embryonic stem cells. Biol Reprod 2003; 68:2150-2156.

6 Hovatta O, Mikkola M, Gertow K, et al. A culture system using human foreskin fibroblasts as feeder cells allows production of human embryonic stem cells. Hum Reprod 2003;18:14041409.

7 Richards M, Tan S, Fong CY, et al. Comparative evaluation of various human feeders for prolonged undifferentiated growth of human embryonic stem cells. Stem Cells, 2003; 21:546-556.

8 Amit M, Carpenter MK, Inokuma MS, et al. Clonally derived human embryonic stem cell lines maintain pluripotency and proliferative potential for prolonged periods of culture. Dev Biol 2000; 227:271-278.

9 Xu C, Inokuma MS, Denham J, et al. Feeder-free growth of undifferentiated human embryonic stem cells. Nat Biotechnol 2001;19:971-974.

10 Ying QL, Nichols J, Chambers I, et al. BMP induction of Id proteins suppresses differentiation and sustains embryonic stem cell self-renewal in collaboration with STAT3. Cell 2003; 115:281- 292.

11 Daheron L, Opitz SL, Zaehres H, et al. LIF/STAT3 signaling fails to maintain self-renewal of human embryonic stem cells. Stem Cells 2004; 22:770-778.

$12 \mathrm{Xu}$ RH, Chen X, Li DS, et al. BMP-4 initiates human embryonic stem cell differentiation to trophoblast. Nat Biotechnol 2002; 20:1261-1264.

13 Pyle AD, Lock LF, Donovan PJ. Neurotrophins mediate human embryonic stem cell survival. Nat Biotechnol. 2006 Mar; 24:34450.

14 Sato N, Meijer L, Skaltsounis L, et al. Maintenance of pluripotency in human and mouse embryonic stem cells through activation of Wnt signaling by a pharmacological GSK-3-specific inhibitor. Nat Med 2004; 10:55-63.

15 Dravid G, Ye Z, Hammond H, et al. Defining the role of Wnt/ beta-catenin signaling in the survival, proliferation and selfrenewal of human embryonic stem cells. Stem Cells 2005; 23: 1489-1501.

16 Amit M, Shariki C, Margulets V, Itskovitz-Eldor J. Feeder layerand serum-free culture of human embryonic stem cells. Biol Reprod. 2004; 70:837-845.

17 Beattie GM, Lopez AD, Bucay N, et al. Activin A maintains pluripotency of human embryonic stem cells in the absence of feeder layers. Stem Cells. 2005, 23:489-495.

18 Xiao L, Yuan X, Sharkis SJ. Activin A maintains self-renewal and regulates fibroblast growth factor, Wnt, and bone morphogenic protein pathways in human embryonic stem cells. Stem Cells. 2006; 24:1476-1486.

19 Xu RH, Peck RM, Li DS et al. Basic FGF and suppression of BMP signaling sustain undifferentiated proliferation of human ES cells. Nat Methods. 2005; 2:185-190.

20 Wang G, Zhang H, Zhao Y, et al. Noggin and bFGF cooperate to maintain the pluripotency of human embryonic stem cells in the absence of feeder layers. Biochem Biophys Res Commun. 2005; 330:934-42.

$21 \mathrm{Xu} \mathrm{C}$, Rosler E, Jiang J, et al. Basic fibroblast growth factor supports undifferentiated human embryonic stem cell growth without conditioned medium. Stem Cells 2005; 23:315-323.

22 Wang L, Li L, Menendez P, Cerdan C, Bhatia M. Human embryonic stem cells maintained in the absence of mouse embryonic fibroblasts or conditioned media are capable of hematopoietic development. Blood. 2005; 105:4598-603.

23 Dravid G, Hammond H. and Cheng L. Culture of human embryonic stem cells on human and mouse feeder cells. In Turksen K, eds Human Embryonic Stem Cells, Totowa: Humana Press, 2004:91-104.

24 Zhou X, Cui Y, Huang X, et al. Lentivirus-Mediated Gene Transfer and Expression in Established Human Cytotoxic T cells Specific to Tumor Antigen and Primary Unstimulated T Cells. Human Gene Therapy 2003; 14:1089-1105.

$25 \mathrm{Xu}$ Q, Wang Y, Dabdoub A, et al. Vascular development in the retina and inner ear: control by Norrin and Frizzled-4, a highaffinity ligand-receptor pair. Cell 2004; 116:883-895.

26 Shaner NC, Campbell RE, Steinbach PA, et al. Improved mono- 
meric red, orange and yellow fluorescent proteins derived from Discosoma sp. red fluorescent protein. Nat Biotechnol 2004; 22:1567-1572.

27 Willert K., Brown JD, Danenberg E, et al. Wnt proteins are lipidmodified and can act as stem cell growth factors. Nature 2003; 423:448-452.

28 Reya T, Duncan AW, Ailles L, et al. A role for Wnt signaling in self-renewal of haematopoietic stem cells. Nature 2003; 423:409414.

29 Moon RT, Kohn AD, De Ferrari GV, et al. WNT and beta-catenin signaling: diseases and therapies. Nat Rev Genet 2004; 5:691701.

30 Nelson WJ, Nusse R. Convergence of Wnt, beta-catenin, and cadherin pathways. Science 2004; 303:1483-1487.

31 Kleber M, Sommer L. Wnt signaling and the regulation of stem cell function. Curr Opin Cell Biol 2004; 16:681-687.

32 Veeman MT, Axelrod JD, Moon RT. A second canon: Functions and mechanisms of $\beta$-catenin-independent Wnt signaling. Dev Cell 2003; 5:367-377.

33 Polesskaya A, Seale P, Rudnicki MA. Wnt signaling induces the myogenic specification of resident CD45+adult stem cells during muscle regeneration. Cell 2003; 113:841-852.

34 Kielman MF, Rindapaa M, Gaspar C, et al. Apc modulates embryonic stem-cell differentiation by modulating the dosage of beta-catenin signaling. Nat Genet 2003; 32: 594-605.

35 Otero JJ, $\mathrm{Fu} \mathrm{W}$, Kan L, et al. Beta-catenin signaling is required for neural differentiation of embryonic stem cells. Development 2004; 131:3545-3557.

36 Huelsken J, Vogel R, Brinkmann V, et al. Requirement for betacatenin in anterior-posterior axis formation in mice. J Cell Biol 2000; 148:567-578.

37 Lee HY, Kleber M, Hari L, et al. Instructive role of Wnt/betacatenin in sensory fate specification in neural crest stem cells. Science 2004; 303:1020-1023.

38 Alonso L, Fuchs E. Stem cells in the skin: waste not, Wnt not. Genes Dev 2003; 17:1189-1200.

39 Tumbar T, Guasch G, Greco V, et al. Defining the epithelial stem cell niche in skin. Science 2004; 303:359-363.

$40 \mathrm{He}$ XC, Zhang J, Tong WG, et al. BMP signaling inhibits intestinal stem cell self-renewal through suppression of Wnt-betacatenin signaling. Nat Genet 2004; 36:1117-1121.

41 Scheller M, Huelsken J, Rosenbauer F, Taketo MM, Birchmeier W, Tenen DG, Leutz A. Hematopoietic stem cell and multilineage defects generated by constitutive beta-catenin activation. Nat Immunol 2006; 7:1037-1047.

42 Kirstetter P, Anderson K, Porse BT, Jacobsen SE, Nerlov C. Activation of the canonical Wnt pathway leads to loss of hematopoietic stem cell repopulation and multilineage differentiation block. Nat Immunol 2006; 7:1048-1056.

43 Trowbridge JJ, Moon RT, Bhatia M. Hematopoietic stem cell biology: too much of a Wnt thing. Nat Immunol 2006; 7:10211023. 